\title{
Comorbidity Assessment in Skin Cancer Patients: A Pilot Study Comparing Medical Interview with a Patient-Reported Questionnaire
}

\author{
Erica H. Lee, ${ }^{1}$ Rajiv I. Nijhawan, ${ }^{1,2}$ Kishwer S. Nehal, ${ }^{1}$ Stephen W. Dusza, ${ }^{1}$ Amanda Levine, ${ }^{3}$ \\ Amanda Hill, ${ }^{3}$ and Christopher A. Barker ${ }^{3}$ \\ ${ }^{1}$ Department of Medicine, Dermatology Service, Memorial Sloan Kettering Cancer Center, New York, NY 10022, USA \\ ${ }^{2}$ Department of Dermatology, University of Texas Southwestern Medical Center, Dallas, TX 75390, USA \\ ${ }^{3}$ Department of Radiation Oncology, Memorial Sloan Kettering Cancer Center, New York, NY 10065, USA \\ Correspondence should be addressed to Erica H. Lee; leee@mskcc.org
}

Received 6 January 2015; Revised 21 May 2015; Accepted 24 May 2015

Academic Editor: Lionel Larue

Copyright (C) 2015 Erica H. Lee et al. This is an open access article distributed under the Creative Commons Attribution License, which permits unrestricted use, distribution, and reproduction in any medium, provided the original work is properly cited.

Background. Comorbidities are conditions that occur simultaneously but independently of another disorder. Among skin cancer patients, comorbidities are common and may influence management. Objective. We compared comorbidity assessment by traditional medical interview (MI) and by standardized patient-reported questionnaire based on the Adult Comorbidity Evaluation27 (ACE-27). Methods. Between September 2011 and October 2013, skin cancer patients underwent prospective comorbidity assessment by a Mohs surgeon (MI) and a radiation oncologist (using a standardized patient-reported questionnaire based on the ACE-27, the PRACE-27). Comorbidities were identified and graded according to the ACE-27 and compared for agreement. Results. Forty-four patients were evaluated. MI and PRACE-27 identified comorbidities in $79.5 \%$ and $88.6 \%(p=0.12)$ of patients, respectively. Among 27 comorbid ailments, the MI identified 9.9\% as being present, while the PRACE-27 identified $12.5 \%$. When there were discordant observations, PRACE-27 was more likely than MI to identify the comorbidity $(\mathrm{OR}=5.4,95 \% \mathrm{CI}=2.4-$ $14.4, p<0.001)$. Overall comorbidity scores were moderate or severe in $43.2 \%(\mathrm{MI})$ versus 59.1\% (PRACE-27) $(p=0.016)$. Limitations. Small sample size from a single institution. Conclusion. Comorbidities are common in skin cancer patients, and a standardized questionnaire may better identify and grade them. More accurate comorbidity assessments may help guide skin cancer management.

\section{Background}

Skin cancer is the most common malignancy worldwide. Management recommendations rely primarily on characteristics of the cancer and patient. One important patient characteristic is comorbidity or the simultaneous presence of a medical condition independent of skin cancer. Comorbidities have been shown to predict outcomes such as infection rates, quality of life, life expectancy, and complications in cancer management, with a trend indicating inferior outcomes in individuals with additional comorbidities [1-5]. Comorbidity severity has been shown to guide treatment selection in a range of conditions from oral cancer to end-stage renal disease [6-9].
A method for assessing comorbidity in a standardized fashion could be valuable in the management of skin cancer patients; however, there are no specific tools for comorbidity evaluation for this population. The Charlson Comorbidity Index (CCI), a validated measure to predict survival, is frequently cited in publications [10]. A recent study evaluated comorbidities in patients $\geq 90$ years old treated with Mohs micrographic surgery (MMS) and found no significant difference in survival among patients with and without comorbidities [11]. On the contrary, another study assessed patients $\geq 80$ years old who underwent dermatologic surgery and found that comorbidities were associated with increased mortality [12]. Similarly, a study of those 90-99 years of age treated with Mohs surgery reported that patients with CCI scores of zero 
(no comorbidities) had longer survival compared to patients with CCI of three (advanced comorbidities) [13].

In addition to the CCI, there are several validated tools used to assess comorbidity such as the Kaplan-Feinstein Index, Adult Comorbidity Evaluation-27 (ACE-27), and the Elixhauser Comorbidity Measure [14-18]. In the general cancer population, the ACE-27 is a validated instrument used to identify and grade 27 comorbidities among nine organ systems plus substance abuse, obesity, and malignancy [14]. It was developed through modification of the Kaplan-Feinstein Comorbidity Index and validated in 19,268 cancer patients treated at Barnes-Jewish Hospital [5]. Studies have shown that the ACE-27 can detect comorbidities more often than the CCI [19-21] and also led to deviations from standard management in patients with comorbidities that were detected by the ACE$27[9,22]$.

In this pilot study, we sought to compare comorbidity assessments collected by the traditional medical interview (MI) in a dermatologic surgery practice and by a standardized patient-reported questionnaire based on the ACE-27 (referred to as PRACE-27 in this paper) ascertained by a radiation oncologist. We specifically used ACE-27 given its validation in a variety of cancer populations, its comprehensive approach, and its application using retrospective note review by cancer registrars $[23,24]$. The objectives of this pilot study were to (1) assess the frequency, severity, and type of comorbidities among skin cancer patients presenting for outpatient management and (2) identify discrepancies in comorbidity assessment between MI and PRACE-27.

\section{Methods}

IRB exemption was obtained at Memorial Sloan Kettering Cancer Center. All patients with a biopsy proven skin cancer evaluated by both a radiation oncologist (CB) and one of two dermatologic surgeons (KN, EL) within a six month period between September 2011 and October 2013 were identified. Comorbidities were ascertained in a prospective manner using one of two techniques. The Mohs surgeons characterized comorbidities through a traditional medical interview (MI). The radiation oncologist performed comorbidity assessment by a patient-reported questionnaire based on the ACE-27 (referred to as PRACE-27).

Medical records were retrospectively reviewed and comorbidities were identified and graded according to the ACE-27. Individual organ systems and diseases were assessed based on specific criteria outlined by the grading guidelines on the following scale: $0=$ no disease, grade $1=$ mild decompensation, grade $2=$ moderate decompensation, and grade 3 = severe decompensation. Based on the grading of the individual organ systems, an overall comorbidity score was calculated on a scale from 0 (no comorbidity) to 3 (severe comorbidity).

2.1. Statistical Analysis. The distributions of assessments for the twenty-seven comorbidities were evaluated for MI and PRACE-27. Cross-classifications between MI and PRACE27 were completed to assess comorbidity prevalence, percent agreement, kappa, and weighted kappa. The kappa $(\kappa)$ value interpretations were graded as poor $(\kappa<0.2)$, fair $(\kappa=0.2-$ $0.4)$, moderate $(\kappa=0.41-0.6)$, substantial $(\kappa=0.61-0.8)$, or excellent $(\kappa=0.81-1.0)$. McNemar's Chi-square and odds ratios with $95 \%$ confidence intervals were estimated for the paired observations. Trends in survival were also assessed. All statistical analyses were performed with Stata v.12.1, Stata Corporation, College Station, TX.

\section{Results}

Forty-four patients were studied. Patient demographic, cancer, and comorbidity data are listed in Table 1. Overall, $79.5 \%$ and $88.6 \%(p=0.12)$ of patients were identified as having a comorbidity, according to the MI and PRACE27 , respectively. The most common comorbid ailments were hypertension, solid tumor, respiratory disease, and cardiac arrhythmia. Overall comorbidity scores were moderate or severe (scores of 2 or 3 ) in $43.2 \%$ (MI) versus 59.1\% (PRACE27 ) of patients, and this difference was statistically significant $(p=0.016$; difference in paired proportions $=0.159(95 \% \mathrm{CI}$ : $0.028-0.29))$.

As noted in Table 2, percent agreement and kappa scores for comorbidity identification in comorbid ailments between the MI and PRACE-27 were excellent (96\%, range: 77.3-100\%, kappa $=0.81$. Among the comorbid ailments assessed by the ACE-27, 9.9\% were identified using MI, while $12.5 \%$ were identified using the PRACE-27. When there were discordant observations, PRACE-27 was more likely than MI to identify the comorbidity $(\mathrm{OR}=5.4,95 \% \mathrm{CI}=2.4-14.4, p<0.001)$. The respiratory system had the lowest percent agreement $(94.7 \%)$, and, for this organ system, 10 patients were graded at a higher severity with the PRACE-27 compared to the MI. Table 3 presents the percent agreement and kappa between MI and PRACE-27 assessments for data grouped by organ system.

The PRACE-27 identified 4 patients (9\%) with one or more comorbidities (related to respiratory disease, obesity, and gastrointestinal disease) that the MI identified as having no comorbidity, compared to 3 patients $(6.8 \%)$ identified by the MI (related to solid organ malignancy) that was missed by the PRACE-27 ( $p=0.38)$. PRACE-27 identified a greater severity of comorbidity when both assessments agreed to its presence. PRACE-27 rated 8 patients with a higher severity than the MI, whereas MI did not increase the severity score for any patients compared to PRACE-27. While there were discrepancies in the comorbidity grading severity of individual organ systems, the agreement in the overall comorbidity score for the patients was high $(91.7 \%$, $p<0.001$ ) (Table 4).

The median follow-up for the 44 patients was 1.9 years from the first comorbidity assessment to the most recent follow-up. There were 7 deaths of which $5(71.4 \%)$ were graded by the PRACE-7 as severe (i.e., 3 ) and 3 (42.8\%) were graded by the MI as severe. Across comorbidity groups, there was a trend for lower survival for higher comorbidities that was significant for both the PRACE-7 and the MI groups $(p<0.05)$. Two-year survival rates were estimated as $100 \%$, 
TABLE 1: Patient demographic data, lesion information, and comorbidity data.

\begin{tabular}{|c|c|c|c|c|c|c|c|c|}
\hline \multicolumn{9}{|c|}{ Demographic data } \\
\hline Average age in years (median; range) & \multicolumn{8}{|c|}{$73.5(76 ; 25-94)$} \\
\hline Female & \multicolumn{8}{|c|}{$22(50.0 \%)$} \\
\hline Male & \multicolumn{8}{|c|}{$22(50.0 \%)$} \\
\hline \multicolumn{9}{|c|}{ Diagnoses } \\
\hline Basal cell carcinoma & \multicolumn{8}{|c|}{$22(50.0 \%)$} \\
\hline Squamous cell carcinoma & \multicolumn{8}{|c|}{$12(27.3 \%)$} \\
\hline Melanoma & \multicolumn{8}{|c|}{$7(15.9 \%)$} \\
\hline Dermatofibrosarcoma protuberans & \multicolumn{8}{|c|}{$1(2.3 \%)$} \\
\hline Merkel cell carcinoma & \multicolumn{8}{|c|}{$1(2.3 \%)$} \\
\hline Microcystic adnexal carcinoma & \multicolumn{8}{|c|}{$1(2.3 \%)$} \\
\hline \multicolumn{9}{|c|}{ Comorbidity data MI versus PRACE-27 } \\
\hline & \multicolumn{4}{|c|}{ MI } & \multicolumn{4}{|c|}{ PRACE-27 } \\
\hline & 0 & 1 & 2 & 3 & 0 & 1 & 2 & 3 \\
\hline \multicolumn{9}{|l|}{ Comorbid ailment } \\
\hline Myocardial infarction & 43 & 1 & - & - & 40 & 2 & 2 & - \\
\hline Angina/coronary artery disease & 38 & 6 & - & - & 37 & 5 & 2 & - \\
\hline Congestive heart failure & 44 & - & - & - & 42 & - & 2 & - \\
\hline Arrhythmias & 36 & - & 8 & - & 35 & 1 & 8 & - \\
\hline Hypertension & 22 & 22 & - & - & 19 & 20 & 5 & - \\
\hline Venous disease & 40 & 2 & 1 & 1 & 40 & 1 & 2 & 1 \\
\hline Peripheral arterial disease & 44 & - & - & - & 42 & - & 2 & - \\
\hline Respiratory system & 42 & 1 & 1 & - & 34 & 6 & 2 & 2 \\
\hline Hepatic & 44 & - & - & - & 43 & - & - & 1 \\
\hline Stomach/intestine & 44 & - & - & - & 44 & 一 & - & - \\
\hline Pancreas & 44 & - & - & - & 44 & - & - & - \\
\hline End-stage renal disease & 44 & - & - & - & 44 & - & - & - \\
\hline Diabetes mellitus & 39 & 3 & 2 & - & 39 & 2 & 1 & 2 \\
\hline Stroke & 39 & 3 & 2 & - & 37 & 6 & 1 & - \\
\hline Dementia & 41 & 2 & - & 1 & 41 & 1 & 1 & 1 \\
\hline Paralysis & 43 & 1 & - & - & 41 & 2 & - & 1 \\
\hline Neuromuscular & 41 & 2 & - & 1 & 40 & 2 & - & 2 \\
\hline Psychiatric & 42 & 2 & - & - & 42 & 1 & 1 & - \\
\hline Rheumatologic & 44 & - & - & - & 43 & 1 & - & - \\
\hline AIDS & 44 & - & - & - & 44 & 一 & - & - \\
\hline Solid tumor including melanoma & 28 & 9 & 6 & 1 & 31 & 7 & 4 & 2 \\
\hline Leukemia and myeloma & 42 & 1 & 1 & - & 42 & 1 & 1 & - \\
\hline Lymphoma & 41 & 3 & - & - & 41 & 2 & - & 1 \\
\hline Alcohol & 44 & - & - & - & 44 & - & - & - \\
\hline Illicit drugs & 44 & - & - & - & 44 & - & - & - \\
\hline Obesity & 44 & - & - & - & 41 & - & 3 & - \\
\hline Overall comorbidity score & 9 & 16 & 12 & 7 & 5 & 13 & 13 & 13 \\
\hline
\end{tabular}

The comorbidity data section compares the number of patients identified as having a comorbid ailment between the MI and PRACE-27. Individual organ systems and diseases were assessed based on specific criteria outlined by the grading guidelines on the following scale: $0=$ no disease, grade $1=$ mild decompensation, grade 2 = moderate decompensation, and grade 3 = severe decompensation. Based on the grading of these individual organ systems, an overall comorbidity score was calculated on a scale from 0 (no comorbidity) to 3 (severe comorbidity).

$92 \%, 91 \%$, and $68 \%$ for grades $0,1,2$, and 3 using the PRACE27. Two-year survival rates were $100 \%, 86 \%, 91 \%$, and $57 \%$ for grades $0,1,2$, and 3 using the MI. These estimations are relatively similar to previously published estimations using the ACE-27: grade 0 (83\%), grade 1 (80\%), grade $2(72 \%)$, and grade 3 (66\%) [5].

\section{Comment}

The National Comprehensive Cancer Network (NCCN) emphasizes the importance of collecting and measuring comorbidities in cancer patients to guide medical care and for research. For skin cancer patients, comorbidities are 
TABLE 2: Comorbid ailments captured and missed by medical interview (MI) and PRACE-27 in individual organ systems.

\begin{tabular}{cccc}
\hline & \multicolumn{2}{c}{ Medical interview } & \multirow{2}{*}{ Total } \\
& Present & Absent & \\
\hline PRACE-27 & & & \\
Present & 111 & $\mathbf{3 8}$ & 149 \\
Absent & 7 & 1032 & 1039 \\
Total & 118 & 1070 & 1188 \\
\hline
\end{tabular}

There are a total of 1,188 possibilities for comorbid ailments to be identified among the forty-four patients in this study since each patient can potentially have twenty-seven comorbidities $(44 \times 27=1,188)$. Both MI and PRACE-27 agreed upon 111 comorbidities being present and 1032 being absent. However, PRACE-27 identified 38 comorbidities that were deemed as being absent by MI while MI identified 7 comorbidities that were deemed absent by PRACE27 [OR $=5.4,95 \%$ CI: $2.4-14.4, p<0.001]$.

TABLE 3: Percent agreement and kappa between medical interview (MI) and PRACE-27 assessments for data grouped by disease system and overall comorbidity scores.

\begin{tabular}{lccc}
\hline Organ system & Percent agreement & Kappa & $p$ value \\
\hline Cardiovascular & 98.7 & 0.80 & $<0.001$ \\
Respiratory & 94.7 & 0.40 & $<0.001$ \\
Gastrointestinal & 99.2 & - & - \\
Renal & 100 & - & - \\
Endocrine & 98.7 & 0.84 & $<0.001$ \\
Neurologic & 98.8 & 0.77 & $<0.001$ \\
Psychiatric & 96.0 & -0.04 & 0.62 \\
Rheumatologic & 97.7 & - & - \\
Immunological & 100 & - & - \\
Malignancy & 98.7 & 0.84 & $<0.001$ \\
Substance abuse & 100 & - & - \\
Obesity & 100 & - & - \\
\hline
\end{tabular}

most commonly assessed using standard medical interview (MI) and rarely graded with a standardized approach. We sought to compare comorbidity collection between MI and a standardized patient-reported questionnaire based on the ACE-27 (PRACE-27).

Identification and agreement of comorbidities across 27 ailments as they relate to individual organ systems were high between the two data collection methods; however, PRACE27 was more likely than MI to identify the existence of an ailment and determine that an ailment was of a greater severity. However, MI appeared to identify solid tumor malignancies more frequently, suggesting that additional attention to capture this ailment may be needed when using the PRACE-27. Nevertheless, the systematic collection of comorbidities with a questionnaire (e.g., PRACE-27) ensures that all organ systems are comprehensively queried. Severity grading may also provide a more accurate assessment of disease status, the overall picture of a patient's health, and potential survival status. Based on this pilot study, a standardized patient-reported questionnaire may better identify and grade individual comorbidities in skin cancer patients compared to standard MI. The concordance in the overall comorbidity score, which is most commonly used to predict outcomes, was also high, suggesting that the PRACE-27 is an accurate assessment of comorbidities, compared to the goldstandard MI [25].

Comorbidity assessment using ACE-27 has been reported to be predictive of treatment modifications. In a study of cancer patients evaluated by a radiation oncologist, a change in treatment plan was noted for patients with moderate or severe indices more often than those with none or mild indices, while age had no contribution to predicting treatment change [22]. Comorbidity grading may similarly guide multidisciplinary management in the skin cancer population. Importantly, in skin cancer populations, patients with less comorbidity have also been shown to have better skin-related quality of life after treatment of both non-melanoma skin cancer (NMSC) and localized melanoma [26, 27]. However, the data is limited in the skin cancer population and additional research is needed to assess whether comorbidities affect outcomes and if systematic assessment should be included in skin cancer management algorithms.

There are a few limitations to this study. First, the number of patients studied was small and the follow-up period was limited to only 2 years. However, the power of the analysis is derived from the large number of binary endpoints used in the comparative analyses. Moreover, this pilot study required cotemporaneous evaluation by a small number of staff from two clinical services, thus limiting the number of potential patients eligible for study. Second, a single institution setting may not be representative of the general skin cancer population. Nevertheless, a study goal was to compare two methods of data collection, and identifying patients evaluated at a single center facilitated this objective. Third, comorbidity data extraction used information from an outpatient clinical assessment and did not utilize review of all of a patient's medical records. While this limited amount of clinical data may have led to underestimation of a patient's comorbid conditions, because most patients with skin cancer receive care by outpatient specialists, this is representative of general clinical practice. Moreover, retrospective chart review has previously been shown to provide sufficient information for comorbidity scoring using ACE-27 with minimal limitations $[23,24]$. Finally, some might suggest that the results of the present study cannot be generalized because of the inability to implement a patient-reported comorbidity assessment in clinical practice. Although there are 27 comorbidities to identify and grade in the PRACE-27, there is a quick learning curve and computing severity scores takes less than three minutes [22].

Comorbidity assessment will likely become an important factor in individualizing treatment for all malignant diseases including skin cancer. It is well accepted that a single treatment approach should not be applied to all patients with skin cancer [28], and thus grading comorbidity severity in patients may help guide management. This pilot study shows that PRACE-27 was more likely to identify an ailment than the MI and better identified the higher grades. The ACE27 is a validated tool that may be more applicable in the skin cancer population compared to other tools such as the Charlson Comorbidity Index (CCI), whose applicability to 
TABLE 4: Percent agreement and kappa between medical interview (MI) and PRACE-27 assessments for overall comorbidity scores.

\begin{tabular}{lcccccc}
\hline & \multicolumn{9}{c}{ PRACE-27 } & Percent agreement & Kappa & $p$ value \\
\hline Overall comorbidity score & 0 & 1 & 2 & 3 & Total \# patients \\
0 & $5^{*}$ & 0 & 0 & 0 & 5 & $<1.7$ \\
1 & $2^{* *}$ & $11^{*}$ & 0 & 0 & 13 & 0.64 \\
2 & $1^{* *}$ & $2^{* *}$ & $9^{*}$ & $1^{* * *}$ & 13 & 13 \\
3 & $1^{* *}$ & $3^{* *}$ & $3^{* *}$ & $6^{*}$ & 44 & 0.01 \\
Total \# patients & 9 & 16 & 12 & 7 & & \\
\hline
\end{tabular}

PRACE-27 and MI had identical overall comorbidity scores for 31/44 patients (marked with $*$ ). There were 12 patients for whom PRACE-27 had a higher overall comorbidity score (marked with $* *$ ) compared to MI and 1 patient (marked with $* * *$ ) for whom MI had a higher overall comorbidity score compared to PRACE-27.

skin cancer populations has been challenged [28]. Utilizing a patient-reported questionnaire like the PRACE-27 during the physician encounter may ensure that important components of the relevant medical history are not omitted. Further research is needed to better delineate the role of comorbidity assessment in the skin cancer population.

\section{Ethical Approval}

IRB exemption was obtained from MKSCC IRB.

\section{Disclosure}

Erica H. Lee and Rajiv I. Nijhawan are co-first authors. Dr. Christopher Barker serves as a paid consultant to Nucletron. MSKCC receives research funding from Nucletron for a study that Dr. Christopher Barker is leading. Nucletron was not involved in this study, in design or through financial support. No other authors have financial disclosure.

\section{Conflict of Interests}

The authors declare that there is no conflict of interests regarding the publication of this paper.

\section{References}

[1] P. C. Albertsen, P. C. Albertsen, D. G. Fryback et al., "The impact of co-morbidity on life expectancy among men with localized prostate cancer," Journal of Urology, vol. 156, no. 1, pp. 127-132, 1996.

[2] R. Yancik, M. N. Wesley, L. A. G. Ries, R. J. Havlik, B. K. Edwards, and J. W. Yates, "Effect of age and comorbidity in postmenopausal breast cancer patients aged 55 years and older," The Journal of the American Medical Association, vol. 285, no. 7, pp. 885-892, 2001.

[3] D. C. Miller, D. A. Taub, R. L. Dunn, J. E. Montie, and J. T. Wei, "The impact of co-morbid disease on cancer control and survival following radical cystectomy, Journal of Urology, vol. 169, no. 1, pp. 105-109, 2003.

[4] C. K. Wells, J. K. Stoller, A. R. Feinstein, and R. I. Horwitz, "Comorbid and clinical determinants of prognosis in endometrial cancer," Archives of Internal Medicine, vol. 144, no. 10, pp. 2004-2009, 1984.
[5] J. F. Piccirillo, R. M. Tierney, I. Costas, L. Grove, and E. L. Spitznagel Jr., "Prognostic importance of comorbidity in a hospital-based cancer registry," Journal of the American Medical Association, vol. 291, no. 20, pp. 2441-2447, 2004.

[6] C.-Y. Ma, T. Ji, A. Ow et al., "Surgical site infection in elderly oral cancer patients: is the evaluation of comorbid conditions helpful in the identification of high-risk ones?" Journal of Oral and Maxillofacial Surgery, vol. 70, no. 10, pp. 2445-2452, 2012.

[7] T. T. A. Peters, B. A. C. van Dijk, J. L. N. Roodenburg, B. F. A. M. van der Laan, and G. B. Halmos, "Relation between age, comorbidity, and complications in patients undergoing major surgery for head and neck cancer," Annals of Surgical Oncology, vol. 21, no. 3, pp. 963-970, 2014.

[8] I. K. Lægreid, K. Aasarod, A. Bye, T. Leivestad, and M. Jordhoy, "The impact of nutritional status, physical function, comorbidity and early versus late start in dialysis on quality of life in older dialysis patients," Renal Failure, vol. 36, no. 1, pp. 9-16, 2014.

[9] G. Baijal, T. Gupta, C. Hotwani et al., "Impact of comorbidity on therapeutic decision-making in head and neck cancer: audit from a comprehensive cancer center in India," Head and Neck, vol. 34, no. 9, pp. 1251-1254, 2012.

[10] M. E. Charlson, P. Pompei, K. A. Ales, and C. R. MacKenzie, "A new method of classifying prognostic comorbidity in longitudinal studies: development and validation," Journal of Chronic Diseases, vol. 40, no. 5, pp. 373-383, 1987.

[11] A. Delaney, I. Shimizu, L. H. Goldberg, and D. F. MacFarlane, "Life expectancy after Mohs micrographic surgery in patients aged 90 years and older," Journal of the American Academy of Dermatology, vol. 68, no. 2, pp. 296-300, 2013.

[12] J. C. Pascual, I. Belinchon, and J. M. Ramos, "Mortality after dermatologic surgery for nonmelanoma skin cancer in patients aged 80 years and older," Journal of the American Academy of Dermatology, vol. 69, no. 6, pp. 1051-1052, 2013.

[13] A. J. Charles Jr., C. C. Otley, and G. R. Pond, "Prognostic factors for life expectancy in nonagenarians with nonmelanoma skin cancer: implications for selecting surgical candidates," Journal of the American Academy of Dermatology, vol. 47, no. 3, pp. 419$422,2002$.

[14] V. Paleri and R. G. Wight, "A cross-comparison of retrospective notes extraction and combined notes extraction and patient interview in the completion of a comorbidity index (ACE-27) in a cohort of United Kingdom patients with head and neck cancer," Journal of Laryngology and Otology, vol. 116, no. 11, pp. 937-941, 2002. 
[15] M. T. A. Sharabiani, P. Aylin, and A. Bottle, "Systematic review of comorbidity indices for administrative data," Medical Care, vol. 50, no. 12, pp. 1109-1118, 2012.

[16] B. S. Linn, M. W. Linn, and L. Gurel, "Cumulative illness rating scale," Journal of the American Geriatrics Society, vol. 16, no. 5, pp. 622-626, 1968.

[17] M. S. Litwin, S. Greenfield, E. P. Elkin, D. P. Lubeck, J. M. Broering, and S. H. Kaplan, "Assessment of prognosis with the total illness burden index for prostate cancer: aiding clinicians in treatment choice," Cancer, vol. 109, no. 9, pp. 1777-1783, 2007.

[18] M. H. Kaplan and A. R. Feinstein, "The importance of classifying initial comorbidity in evaluating the outcome of diabetes mellitus," Journal of Chronic Diseases, vol. 27, no. 7-8, pp. 387404, 1974.

[19] R. Mayr, M. May, T. Martini et al., "Predictive capacity of four comorbidity indices estimating perioperative mortality after radical cystectomy for urothelial carcinoma of the bladder," $B J U$ International, vol. 110, no. 6, pp. E222-E227, 2012.

[20] D. Kallogjeri, S. M. Gaynor, M. L. Piccirillo, R. A. Jean, E. L. Spitznagel Jr., and J. F. Piccirillo, "Comparison of comorbidity collection methods," Journal of the American College of Surgeons, vol. 219, no. 2, pp. 245-55, 2014.

[21] V. S. Nesic, Z. M. Petrovic, S. B. Sipetic, S. D. Jesic, I. A. Soldatovic, and D. A. Kastratovic, "Comparison of the Adult Comorbidity Evaluation 27 and the Charlson Comorbidity indices in patients with laryngeal squamous cell carcinoma," Journal of Laryngology and Otology, vol. 126, no. 5, pp. 516-524, 2012.

[22] J. B. Owen, N. Khalid, A. Ho et al., "Can patient comorbidities be included in clinical performance measures for radiation oncology?", Journal of Oncology Practice, vol. 10, no. 3, pp. el75e181, 2014.

[23] V. Paleri and R. G. Wight, "Applicability of the adult comorbidity evaluation-27 and the Charlson indexes to assess comorbidity by notes extraction in a cohort of United Kingdom patients with head and neck cancer: a retrospective study," Journal of Laryngology and Otology, vol. 116, no. 3, pp. 200-205, 2002.

[24] J. F. Piccirillo, C. M. Creech, R. Zequeira, S. Anderson, and A. S. Johnston, "Inclusion of comorbidity into oncoogy data registries," Journal of Registry Management, vol. 26, pp. 66-70, 1999.

[25] N. Daver, K. Naqvi, E. Jabbour et al., "Impact of comorbidities by ACE-27 in the revised-IPSS for patients with myelodysplastic syndromes," American Journal of Hematology, vol. 89, no. 5, pp. 509-516, 2014.

[26] T. Chen, D. Bertenthal, A. Sahay, S. Sen, and M.-M. Chren, "Predictors of skin-related quality of life after treatment of cutaneous basal cell carcinoma and squamous cell carcinoma," Archives of Dermatology, vol. 143, no. 11, pp. 1386-1392, 2007.

[27] G. Schubert-Fritschle, A. Schlesinger-Raab, R. Hein et al., "Quality of life and comorbidity in localized malignant melanoma: results of a German population-based cohort study," International Journal of Dermatology, vol. 52, no. 6, pp. 693-704, 2013.

[28] J. F. Sobanko and S. T. Ross, "Mistaken conclusions in a nonmelanoma skin cancer article published in JAMA," Dermatologic Surgery, vol. 40, no. 5, pp. 489-496, 2014. 


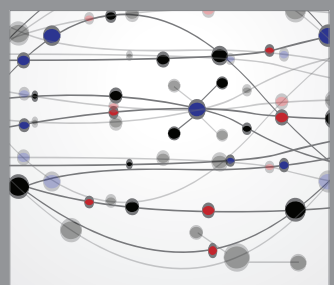

The Scientific World Journal
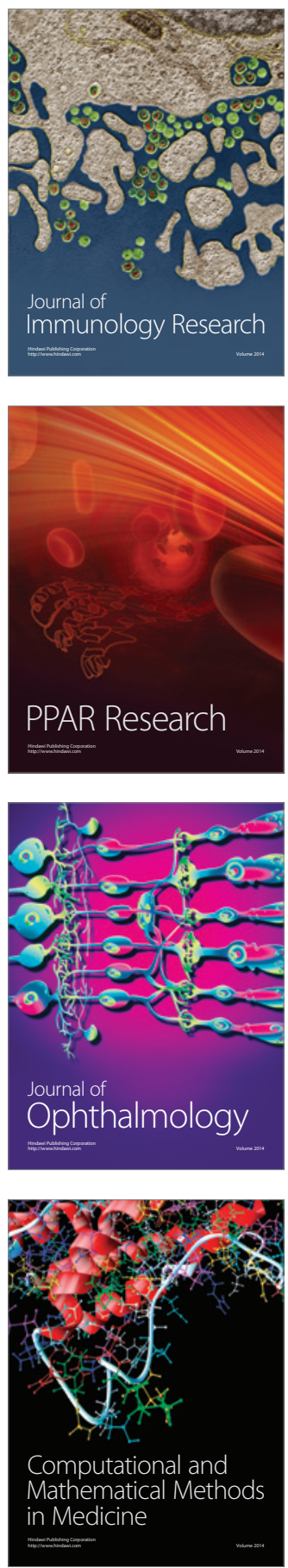

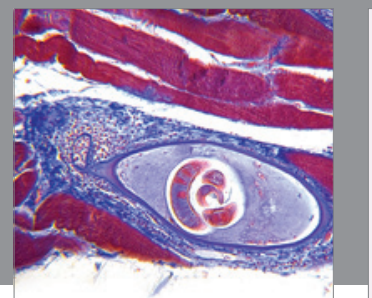

Gastroenterology

Research and Practice
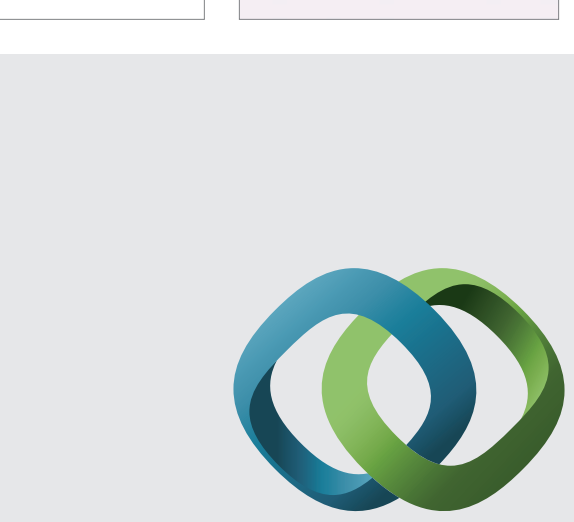

\section{Hindawi}

Submit your manuscripts at

http://www.hindawi.com
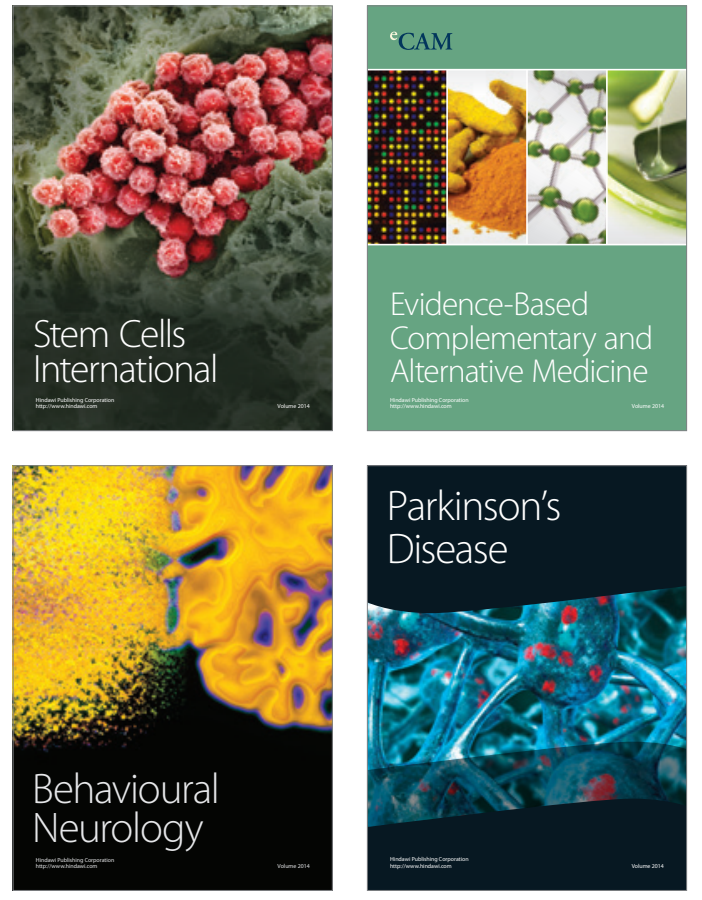
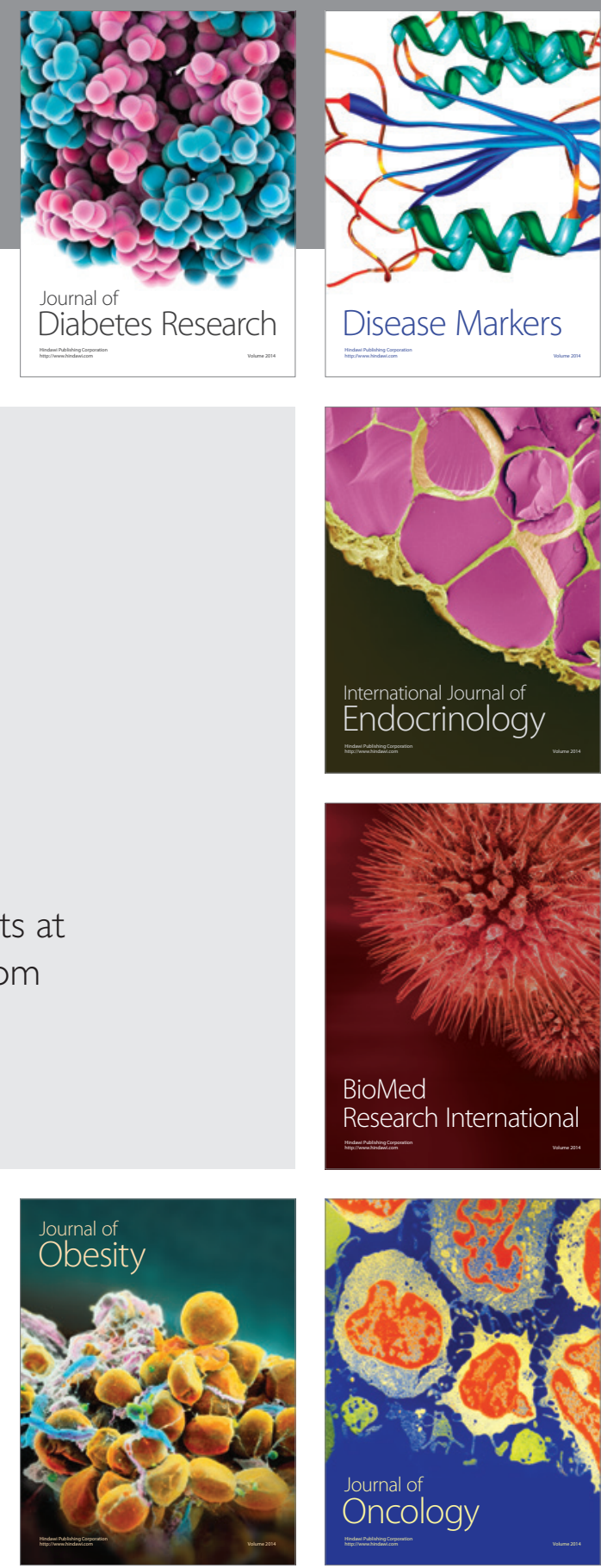

Disease Markers
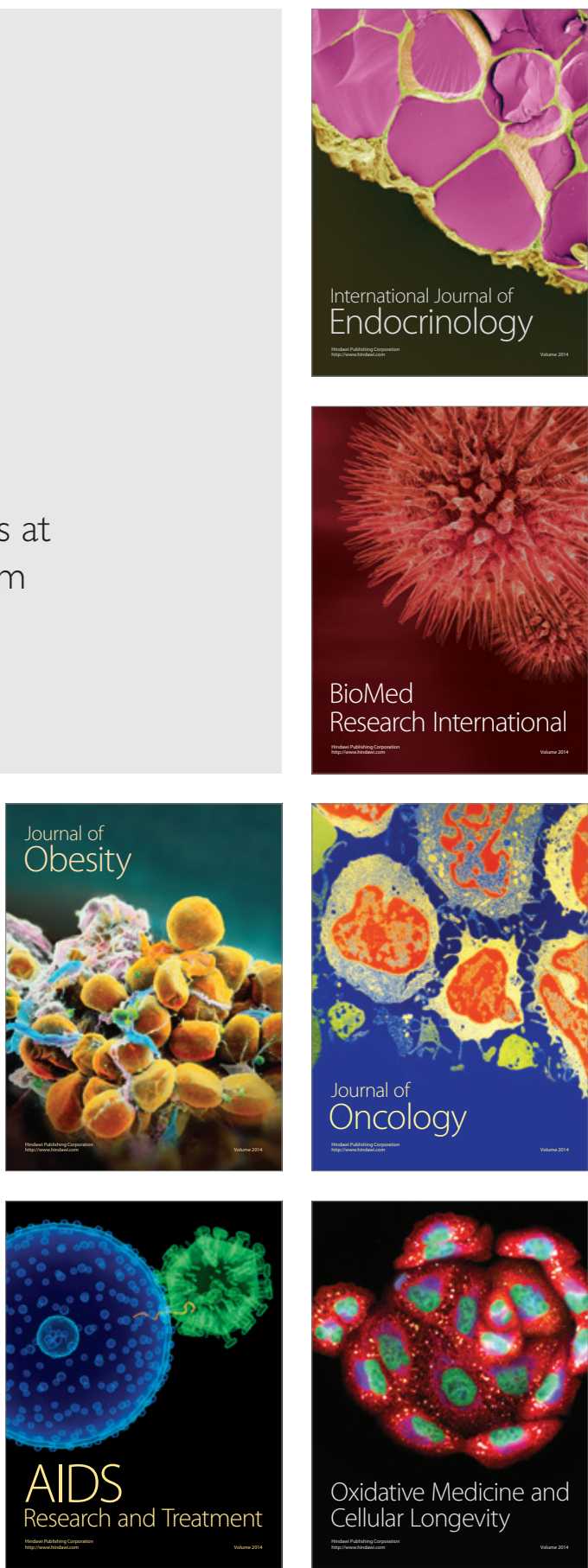